\title{
Evaluation of efficacy and safety of minimally invasive segmentectomy in the treatment of lung cancer
}

\author{
CHANGQING SHAO $^{1}$, CUILING ZHENG $^{2}$, WANSHU YAN ${ }^{3}$, YI SHEN $^{1}$ and ZHIXUE ZHANG ${ }^{1}$ \\ ${ }^{1}$ Departments of Thoracic Surgery, ${ }^{2}$ Spine Surgery and ${ }^{3}$ Emergency, The Affiliated Central Hospital of \\ Qingdao University, Qingdao, Shandong 266000, P.R. China
}

Received July 19, 2017; Accepted November 14, 2017

DOI: $10.3892 / \mathrm{ol} .2018 .8483$

\begin{abstract}
The aim of the study was to investigate the efficacy and safety of minimally invasive segmentectomy in the treatment of lung cancer. A total of 86 lung cancer patients in early stage were selected for the treatment between May, 2010 and December, 2010. The patients were randomly divided into the control $(n=43)$ and observation $(n=43)$ groups. Patients in the control group received conventional thoracotomy as treatment, while thoracoscopic segmentectomy was performed for patients in the observation group. Factors including intraoperative bleeding amount, number of dissected lymph nodes, surgery duration, postoperative intubation time and length of stay (LOS) were compared between the two groups. A visual analogue scale was used for comparison of the postoperative incisional pain experienced by patients in the two groups. The incidence rate of postoperative complications of patients in the two groups was observed. We also assayed variations in the levels of serum inflammatory factors C-reaction protein (CRP), interleukin (IL) -6 and -10 of patients prior to operation and on the $3 \mathrm{rd}$, 5th and 7 th days and after operation via ELISA, and on the 7th day after operation, we determined the pulmonary function of patients. During the 5-year follow-up, the recurrence and survival rate of patients in the two groups were observed. In the observation group, the intraoperative bleeding amount of patients was significantly lower than that in the control group, and the surgery duration, postoperative intubation time and LOS were all shorter than those in the control group $(\mathrm{P}<0.05)$. By contrast, no significant difference was detected in a comparison of the number of dissected lymph nodes of patients between the two groups $(\mathrm{P}>0.05)$. Additionally, in the observation group, patients suffered less pain after operation than those in the control group $(\mathrm{P}<0.05)$, and on the $3 \mathrm{rd}, 5$ th and 7 th days after
\end{abstract}

Correspondence to: Dr Yi Shen, Department of Thoracic Surgery, The Affiliated Central Hospital of Qingdao University, 6 Jiangsu Road, Qingdao, Shandong 266000, P.R. China

E-mail: yishen0718@163.com

Key words: thoracoscopic segmentectomy, lung cancer, minimally invasive surgery operation, the levels of CRP, IL-6 and -10 in the observation group were significantly lower than those in the control group $(\mathrm{P}<0.05)$. After operation, the incidence rate of complications in the observation group was significantly lower than that in the control group $(\mathrm{P}<0.05)$, and the recovery in pulmonary function after operation was superior to that in the control group $(\mathrm{P}<0.05)$. In addition, the 5 -year survival rate of patients in the observation group was significantly higher than that in the control group, and the recurrence rate was significantly lower than that in the control group $(\mathrm{P}<0.05)$. Minimally invasive segmentectomy shows better efficacy in the treatment of lung cancer at early stage than the conventional thoracotomy. In addition to the high safety during surgery, this technique can lower the incidence rate of postoperative complications, protect the pulmonary function, increase the survival rate and decrease the recurrence rate, which shows great value in clinical practice.

\section{Introduction}

Exposure to tobacco, dietary habits, occupational carcinogens, environmental pollutions and the characteristics of host are factors that have played an increasingly important role in the high lung cancer incidence in at least the last 50 years, making this disease the most common cause for cancer-related mortality (1). In China, the mortality rate of lung cancer ranks first in various types of malignant tumors (2). Nevertheless, the early detection rate of lung cancer is gradually on the increase with the development of early screening techniques of lung cancer and the enhancement in the consciousness of physical examination of residents.

According to the survey of US medical care insurance surveillance systems, no distant metastasis of tumor was identified in the first diagnosis of over $40 \%$ of lung cancer patients (3). Surgery is considered the main method for the treatment of non-small cell lung cancer at the early stage, but the application of conventional thoracotomy is limited by long surgery incision, severe injuries to the thoracic muscle, heavy damage to postoperative pulmonary functions, a variety of complications, which have become the major causes for patients succumbing to the disease during the perioperative period (4). Ever since the application of the thoracoscope for thoracic surgery in the late 20 th century, the minimally invasive technique, with the advantages, including small incision, slight injury and rapid 
Table I. Comparison of the general material of patients between the two groups.

\begin{tabular}{lccc}
\hline Items & Control group $(\mathrm{n}=43)$ & Observation group $(\mathrm{n}=43)$ & $\mathrm{t} / \chi^{2}$ \\
\hline Sex (male/female) & $26 / 17$ & $25 / 18$ & 0.048 \\
Age (years) & $40-70$ & $40-75$ & 0.826 \\
Average age (years) & $56.69 \pm 7.49$ & $55.59 \pm 7.58$ & 0.677 \\
Smoking (n, \%) & & & \\
Cigarettes $\geq 5$ & $21(48.84)$ & $19(44.19)$ & 0.500 \\
Cigarettes $<5$ & $8(18.60)$ & $9(20.93)$ & 0.908 \\
Non-smoker & $14(32.56)$ & $15(34.88)$ &
\end{tabular}

postoperative recovery, has been employed in many research domains of thoracic surgery (5). Furthermore, after the concept of 'Precision Medicine' was suggested, this technique has been widely applied in the treatment of lung cancer at early stage (6).

In the present study, we performed minimally invasive segmentectomy for the treatment of lung cancer at early stage to investigate the clinical safety and efficacy of this technique, thereby providing more appropriate procedures for lung cancer patients at early stage. The results corroborate the efficacy and safety of this technique.

\section{Materials and methods}

General material. We selected 86 lung cancer patients at early stage who were admitted to the Affiliated Central Hospital of Qingdao University (Qingdao, China) for treatment between May 2010 and December 2010. Based on the random number table, the patients were randomly divided into the control $(n=43)$ and observation $(n=43)$ groups. Inclusion criteria for the study were: i) Patients who were diagnosed as having lung cancer at early stage via computed tomography and pathological examination; ii) patients who had not received any prior surgery, chemotherapy or radiotherapy; and iii) patients who provided written informed consent. Exclusion criteria for the study were: i) Patients with abnormal coagulation function or with a clinical stage above stage IIIa; and ii) patients with a history of thoracotomy or metastasis in hilar or mediastinal lymph nodes, or distant metastasis. No statistically significant difference was identified in comparison of the general material of patients between the two groups $(\mathrm{P}>0.05$; Table I). This study was approved by the Ethics Committee of The Affiliated Central Hospital of Qingdao University and informed consents were signed by the patients and/or guardians.

Preoperative preparation. In the two groups, patients received regular examinations prior to surgery to eliminate any surgical contradictions. Two weeks prior to operation, the patients were required to discontinue smoking, fasting was required for patients at $6 \mathrm{~h}$ before operation, and at $4 \mathrm{~h}$ before operation, the patients were required to take $300-400 \mathrm{ml} \mathrm{10 \%}$ glucose injection via drinking or injection.

Treatment. Patients in the two groups received endotracheal intubation for intravenous anesthesia, during which the arterial blood gases, arterial blood pressure, pulse and electrocardiogram were monitored. For patients in the control group, conventional thoracotomy was performed as treatment. With patients in the lateral position, an incision was made on the posterolateral site to expose the thoracic cavity layer by layer. After dissection of the mediastinal lymph nodes, the patients underwent regular lobectomy, during which unnecessary squeeze or pull of normal lung tissues was avoided during surgery. After the operation, the thoracic cavity was rinsed using warm saline, the residual ends of lung and bronchus were examined to identify air leakage, and the drainage tube was left in the cavity followed by suturing the incisions.

In the observation group, patients in the lateral position underwent video-assisted thoracoscopic segmentectomy as treatment. After regular disinfection, the patients were required to lie on the operating drape, and an incision of approximately $1 \mathrm{~cm}$ long was made between the 7th and 8th ribs along the midaxillary line on the affected side as the observation hole, the main operation hole was prepared through the incision (approximately $3 \mathrm{~cm}$ in length) between the 4th and 5th ribs along the anterior axillary line, and the auxiliary operation hole was prepared through the incision (approximately $1 \mathrm{~cm}$ in length) in the 8 th rib along the scapular line. After the thoracoscope was placed, we observed the thoracic cavity, and performed the segmentectomy for different pulmonary segments, such as left superior pulmonary segment, lingual segment, anterior segment of right superior lung, apex and dorsal and posterior segments of right superior lung. Subsequently, mediastinal lymph nodes were dissected and the procedures conducted during the operation were guided via video. Following surgery, we examined the dilation of residual lung, and air leakages in the residual ends of lung and bronchus. The drainage tube was then placed through the observation hole, and the incision was sutured.

Detection of CRP, IL-6 and -10 levels in serum. In the two groups, we extracted 3-5 ml fasting peripheral venous blood in the morning before operation and on the 3rd, 5th and 7 th days after operation. Samples were centrifuged for $15 \mathrm{~min}$ at $1,000 \mathrm{xg}$, and the supernatant was placed in an Eppendorf tube for preservation in a refrigerator at $-20^{\circ} \mathrm{C}$. C-reaction protein (CRP), interleukin (IL) -6 and -10 levels in serum were detected via enzyme-linked immunosorbent assay (ELISA), in which the relevant kits were purchased from Shanghai Haoben Biotechnology Co., Ltd., (Shanghai, China) and all the procedures were in accordance with the manufacturer's instructions. Optical density was read by a microplate reader (Thermo Fisher Scientific, Waltham, MA, USA), with which 
Table II. Comparison of surgery condition of patients between the two groups.

\begin{tabular}{lccrr}
\hline Groups & Observation group $(\mathrm{n}=43)$ & Control group $(\mathrm{n}=43)$ & t-test & P-value \\
\hline Surgery duration (min) & $123.24 \pm 8.65$ & $163.56 \pm 9.72$ & 20.320 & $<0.001$ \\
Intraoperative & $226.48 \pm 108.35$ & $349.58 \pm 107.47$ & 5.289 & $<0.001$ \\
bleeding amount (ml) & $3.47 \pm 1.15$ & & & \\
Intubation time (days) & $7.35 \pm 1.28$ & $6.64 \pm 1.26$ & 12.185 & $<0.001$ \\
Quantity of dissected & $9.35 \pm 3.28$ & $7.68 \pm 1.36$ & 1.159 & 0.250 \\
lymph nodes (n) & & $16.68 \pm 3.36$ & 10.237 & $<0.001$ \\
LOS (days) & & & & \\
\hline
\end{tabular}

Table III. Comparison of postoperative pain rate of patients between the two groups.

\begin{tabular}{lccrc}
\hline Groups & Cases & Grade I & Grade II & Grade III \\
\hline Observation & 43 & $31(72.09)$ & $9(20.93)$ & $3(6.98)$ \\
Control & 43 & $16(37.21)$ & $12(27.91)$ & $11(25.58)$ \\
$\chi^{2}$ & & 13.787 & & $0(0.00)$ \\
P-value & & 0.003 & & $4(9.30)$ \\
\hline
\end{tabular}

the corresponding levels of CRP, IL-6 and IL-10 in samples were calculated.

Postoperative treatment and follow-up. Following surgery, patients in the two groups received regular treatment, such as antibiotics and aerosol inhalation. After the drainage tubes were removed, re-examinations were carried out through the chest X-ray images. After being discharged, the patients received 5-year follow-up as follows: i) Once every 3 months in the first two years; ii) once every 6 months in the 2nd and 3 rd years; and iii) once every year in the 4th and 5 th years. During follow-up, we observed the recurrence and survival rate of patients.

Evaluation indexes. The intraoperative bleeding amount, quantity of dissected lymph nodes, postoperative intubation time and length of stay (LOS) were recorded, and the incidence of postoperative complications, including the recordings of incidence rate of intraoperative bleeding amount, persistent air leakage ( $>1$ week), and postoperative arrhythmia and atelectasis were compared.

The visual analogue scale was used for evaluating the pain of patients within a range of $0-10$ points ( 0 point for no pain, and 10 points for intolerable acute pain), and the evaluation criteria were set as: i) Grade I: score between 0 and 2 points for no or imperceptible pain; ii) grade II: score between 3 and 5 points for tolerable pain; iii) grade III: score between 6 and 8 points for obvious pain that affected regular activities; and iv) grade IV: score $\geq 8$ points for intolerable pain.

Prior to operation and on the 3rd, 5th and 7th days after operation, $5 \mathrm{ml}$ fasting venous blood was collected from the patients for isolation and extraction of serum, and the levels of CRP, IL-6 and -10 in serum were detected via ELISA. Three months after operation, we examined the pulmonary functions of patients, including the indexes: forced vital capacity (FVC), forced expiratory volume in one second (FEV1), FEV1/FVC and maximal expiratory flow rate.

During the 5-year follow-up, we carried out statistics on the recurrence rate, disease-free survival (DFS), and 5-year survival rate, in which the DFS started from the 1st day after the operation and ended at the time of initial recurrence or metastasis. In addition, for patients with no recurrence or metastasis during the 5-year follow-up, this period was considered as 5 years.

Statistical analysis. SPSS 19.0 (IBM Corp., Armonk, NY, USA) was employed for data processing. Measurement data were presented as mean \pm standard deviation (false), and the Kaplan-Meier test was carried out for intergroup comparison. Countable data were presented as rate, and $\chi^{2}$ test was performed. $\mathrm{P}<0.05$ was considered to indicate a statistically significant difference.

\section{Results}

Comparison of surgical condition of patients between the two groups. A comparison of surgical conditions of patients between the two groups showed that the surgery duration, postoperative intubation time and LOS were shorter than those in the control group, and the intraoperative bleeding amount of patients were significantly lower than that in the control group $(\mathrm{P}<0.05)$. However, no significant difference was detected in a comparison of the number of dissected lymph nodes of patients between the two groups $(\mathrm{P}>0.05$; Table II).

Comparison of postoperative pain rate between the two groups. A comparison of the postoperative pain degree of patients between the two groups showed that in the observation group, patients suffered less pain following surgery than those in the control group, with a statistically significant difference $(\mathrm{P}<0.05$; Table III). 
Table IV. Comparison of CRP level in patients between the two groups (mg/l).

\begin{tabular}{|c|c|c|c|c|c|}
\hline Groups & Cases & $\begin{array}{c}\text { Before } \\
\text { operation }\end{array}$ & $\begin{array}{l}\text { On the } 3 \text { rd day } \\
\text { after operation }\end{array}$ & $\begin{array}{l}\text { On the } 5 \text { th day } \\
\text { after operation }\end{array}$ & $\begin{array}{l}\text { On the } 7 \text { th day } \\
\text { after operation }\end{array}$ \\
\hline Observation & 43 & $3.43 \pm 1.24$ & $39.67 \pm 3.23$ & $25.35 \pm 3.28$ & $18.68 \pm 3.24$ \\
\hline Control & 43 & $3.62 \pm 1.58$ & $57.27 \pm 3.64$ & $46.47 \pm 3.36$ & $32.83 \pm 3.78$ \\
\hline t-test & & 0.620 & 23.716 & 29.495 & 18.637 \\
\hline P-value & & 0.537 & $<0.001$ & $<0.001$ & $<0.001$ \\
\hline
\end{tabular}

CRP C-reaction protein.

Table V. Comparison of IL-6 level in patients between the two groups (ng/l).

\begin{tabular}{|c|c|c|c|c|c|}
\hline Groups & Cases & $\begin{array}{c}\text { Before } \\
\text { operation }\end{array}$ & $\begin{array}{l}\text { On the } 3 \text { rd day } \\
\text { after operation }\end{array}$ & $\begin{array}{l}\text { On the } 5 \text { th day } \\
\text { after operation }\end{array}$ & $\begin{array}{l}\text { On the } 7 \text { th day } \\
\text { after operation }\end{array}$ \\
\hline Observation & 43 & $46.43 \pm 3.24$ & $121.67 \pm 7.23$ & $106.35 \pm 5.25$ & $78.67 \pm 3.23$ \\
\hline Control & 43 & $47.62 \pm 3.58$ & $137.27 \pm 7.64$ & $126.45 \pm 5.32$ & $96.82 \pm 3.79$ \\
\hline t-test & & 1.616 & 9.725 & 17.634 & 23.901 \\
\hline P-value & & 0.110 & $<0.001$ & $<0.001$ & $<0.001$ \\
\hline
\end{tabular}

IL-6, interleukin-6.

Table VI. Comparison of IL-10 level in patients between the two groups (ng/l).

\begin{tabular}{|c|c|c|c|c|c|}
\hline Groups & Cases & $\begin{array}{l}\text { Before } \\
\text { operation }\end{array}$ & $\begin{array}{l}\text { On the } 3 \text { rd day } \\
\text { after operation }\end{array}$ & $\begin{array}{l}\text { On the } 5 \text { th day } \\
\text { after operation }\end{array}$ & $\begin{array}{l}\text { On the } 7 \text { th day } \\
\text { after operation }\end{array}$ \\
\hline Observation & 43 & $45.75 \pm 3.25$ & $122.64 \pm 6.28$ & $106.36 \pm 4.27$ & $73.52 \pm 3.16$ \\
\hline Control & 43 & $46.82 \pm 3.57$ & $138.56 \pm 6.63$ & $125.47 \pm 5.36$ & $97.56 \pm 3.74$ \\
\hline t-test & & 1.453 & 11.432 & 18.286 & 32.196 \\
\hline P-value & & 0.150 & $<0.001$ & $<0.001$ & $<0.001$ \\
\hline
\end{tabular}

IL-10, interleukin-10.

Comparison of CRP, IL-6 and-10 level in patients between the two groups. We compared the CRP level of patients between the two groups, and found that before operation, there were no significant differences $(\mathrm{P}>0.05)$. By contrast, on the 3rd, 5th and 7 th days after operation, the CRP levels in the observation group were significantly lower than those in the control group $(\mathrm{P}<0.05$; Table IV).

We compared the IL- 6 levels of patients between the two groups, and found that before operation, there were no significant differences $(\mathrm{P}>0.05)$. By contrast, on the $3 \mathrm{rd}, 5 \mathrm{th}$ and 7 th days after operation, the IL-6 levels in the observation group were significantly lower than those in the control group $(\mathrm{P}<0.05$; Table V).

We compared the IL-10 levels of patients between the two groups, and found that before surgery, there was no significant difference $(\mathrm{P}>0.05)$. By contrast, on the 3rd, 5th and 7 th days after operation, the level of IL-10 in the observation group was significantly lower than those in the control group $(\mathrm{P}<0.05$; Table VI).
Comparison of adverse reactions, postoperative pulmonary functions, and the recurrence and survival rates of patients between the two groups. A comparison of the incidence rate of postoperative complications of patients in the two groups showed that following surgery, the incidence rate of complications [postoperative bleeding amount, persistent air leakage ( $>1$ week), postoperative arrhythmia and atelectasis] in the observation group was $6.98 \%$, which was significantly lower than that in the control group $(30.23 \%)(\mathrm{P}<0.05$; Table VII).

Comparison on postoperative pulmonary functions of patients between the two groups revealed that the FVC, FEV1, FEV1/FVC and PEER in the observation group were all significantly higher than those in the control group $(\mathrm{P}<0.05$; Table VIII).

We compared the recurrence and survival rates of patients between the two groups, and found that the average survival period in the observation group was significantly longer than that in the control group, the recurrence rate was significantly lower than that in the control group, and the postoperative 
Table VII. Comparison of adverse reactions of patients between the two groups (n,\%).

\begin{tabular}{|c|c|c|c|c|c|c|}
\hline Groups & Cases & $\begin{array}{c}\text { Intraoperative } \\
\text { bleeding amount }\end{array}$ & $\begin{array}{c}\text { Persistent } \\
\text { air leakage }\end{array}$ & $\begin{array}{l}\text { Postoperative } \\
\text { arrhythmia }\end{array}$ & Atelectasis & Total \\
\hline Observation & 43 & $1(2.33)$ & $0(0)$ & $1(2.33)$ & $1(2.33)$ & $3(6.98)$ \\
\hline Control & 43 & $4(9.30)$ & $3(6.98)$ & $2(4.65)$ & $4(9.30)$ & $13(30.23)$ \\
\hline$\chi^{2}$ & & 6.202 & & & & \\
\hline P-value & & 0.012 & $<0.001$ & & & \\
\hline
\end{tabular}

Table VIII. Comparison of pulmonary function indexes of patients between the two groups.

\begin{tabular}{|c|c|c|c|c|c|}
\hline Groups & Cases (n) & FEV $1(\%)$ & PEER (1/sec) & FEV1/FVC (\%) & FVC (l) \\
\hline Control & 43 & $66.23 \pm 4.02$ & $3.41 \pm 1.24$ & $2.15 \pm 0.33$ & $1.75 \pm 0.64$ \\
\hline Observation & 43 & $71.75 \pm 4.56$ & $4.26 \pm 1.36$ & $2.56 \pm 0.35$ & $2.19 \pm 0.73$ \\
\hline t-test & & 5.954 & 3.029 & 5.589 & 2.972 \\
\hline P-value & & $<0.001$ & 0.003 & $<0.001$ & 0.004 \\
\hline
\end{tabular}

Table IX. Comparison of 5-year follow-up of patients between the two groups.

\begin{tabular}{|c|c|c|c|c|}
\hline Groups & Cases & $\begin{array}{c}\text { 5-year survival } \\
(\mathrm{n}, \%)\end{array}$ & $\begin{array}{c}\text { Recurrence rate } \\
(\mathrm{n}, \%)\end{array}$ & $\begin{array}{l}\text { Average survival } \\
\text { period (months) }\end{array}$ \\
\hline Observation & 43 & $31(72.09)$ & $2(4.65)$ & $48.56 \pm 7.48$ \\
\hline Control & 43 & $21(48.83)$ & $8(18.60)$ & $41.64 \pm 7.53$ \\
\hline$t / \chi^{2}$ & & 4.864 & 4.074 & 4.152 \\
\hline P-value & & 0.027 & 0.044 & 0.001 \\
\hline
\end{tabular}

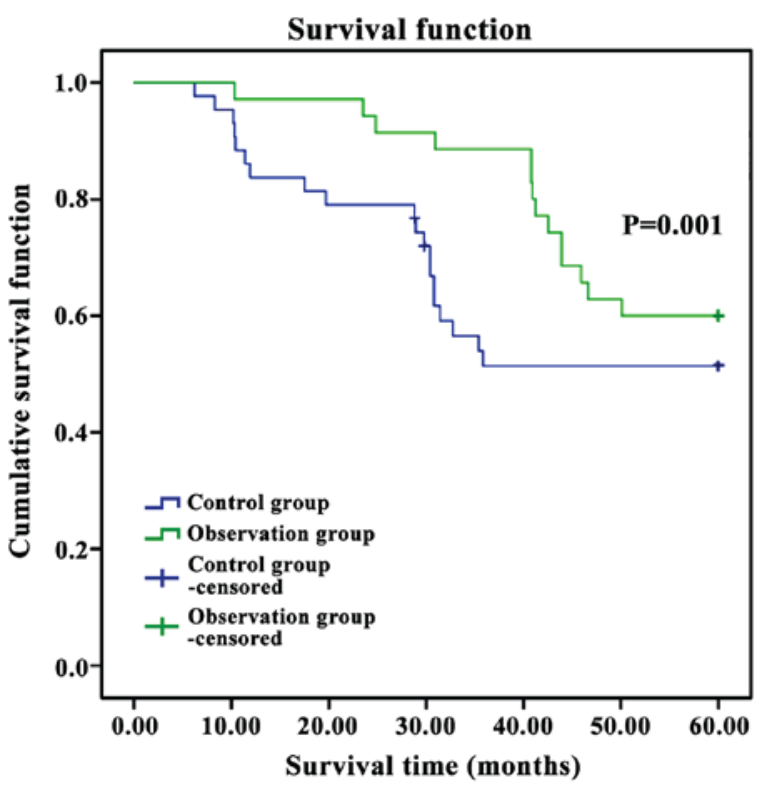

Figure 1. Survival curves of patients in both groups. The Kaplan-Meier analysis revealed that patients in the observation group experienced a significantly longer survival time than that of the control group $(\mathrm{P}<0.05)$.

5-year survival rate was significantly higher than that in the control group ( $\mathrm{P}<0.05$; Table IX and Fig. 1).

\section{Discussion}

The incidence and mortality rates of lung cancer are on the increase worldwide. In many countries, lung cancer has become the primary malignant tumor and poses a threat to the health of human beings (7). The clinical manifestations of lung cancer are not obvious at early stage, but when it comes to advanced stage, symptoms, generally including fever, weight loss, coughing, chest pains, blood-stained sputum, hemoptysis, chest distress and short of breath, emerges at the advanced stage, which are relatively acute in elderly patients (8). At the middle and advanced stages, patients with lung cancer may suffer from direct, lymphatic or hematogenous metastasis, and poor prognosis, leading to a significant decrease in the patient survival rate (9). However, with the enhancement in the consciousness on healthcare and the development in imaging techniques, the detection rate of lung cancer at an early stage is also on the increase. The present and previous findings (10) have shown that the 5-year survival rate of lung cancer patients in stages IA, IB, IIA and IIB who underwent surgery as treatment may be increased from 50 to $80 \%$. Thus, early diagnosis and treatment are of great significance for lung cancer.

Conventional thoracotomy is generally applied in the treatment of lung cancer, but its application has been limited by larger surgical incision, more significant postoperative pain and additional complications (11). In previous years, 
continuous improvement and updates have been observed in surgical and anesthetic techniques and the equipment in thoracic surgeries, and an increasing number of surgical methods have been applied in the treatment of lung cancer, especially the increasingly mature minimally invasive technique (12). At the same time, adverse effects on patients are markedly reduced (12).

The results of the present study showed that duration of surgery, intubation time and LOS of patients in the observation group were significantly shorter than those in the control group, the intraoperative bleeding amount was also significantly lower than that in the control group, and the pain rate of patients in the observation group was significantly reduced when compared with the control group, suggesting that the degree of pain in patients was significantly ameliorated $(\mathrm{P}<0.05)$. Additionally, there was no significant difference in a comparison of the quantity of lymph nodes that were dissected in the operation for the two groups $(\mathrm{P}>0.05)$. This was because in the operation conducted using conventional thoracotomy the major muscular tissues of patients (e.g., the musculus serratus anterior and the latissimus dorsi) were excised, thus enlarging surgical incision, further increasing the intraoperative bleeding amount. By contrast, the rib cage was distracted using a rib retractor, which would generate larger trauma and stimulation to patients, thus significantly aggravating the degree of pain of patients following surgery. However, compared with the conventional thoracotomy, surgeries guided via video provide surgeons with an amplified surgery image through a smaller incision. Consequently, through utilization of video imaging, surgeons were better able to identify the lymph nodes and tiny veins allowing for more efficient isolation and ligation of the microstructures via a smaller incision without any unnecessary damage, and dissect the lymph nodes in deep sites thoroughly, thus reaching similar dissection efficiency with the conventional thoracotomy. Additionally, minimally invasive surgery could save the time for opening and closing the thoracic cavity, allowing for surgery time to be significantly shortened (13).

Surgicaltreatmentmayinduce stressresponses oflung cancer patients, sequentially resulting in the changes in catechol, and the immunosuppression may be induced to activate the large section of cytokines, thus causing inflammatory responses (14). CRP is a kind of acute phase reactive protein, and usually serves as an index for inflammatory responses in clinical practice. By binding chromatin, it can activate the complement system to enhance the leukocyte phagocytic function, and exert a regulatory effect by activating the cells (15). Under normal circumstances, the content of CRP is usually very low, but when it comes to injuries, its concentration is markedly increased. Thus, it can be frequently detected in patients after operation (16). As a member of hte interleukin family, IL-6 is a kind of reactive lymphocyte factor in the acute phase and plays diversified roles in immune responses (17). Stress responses in the surgeries of lung cancer affect the activities of macrophages and $\mathrm{T}$ cells, thus promoting the secretion and generation of IL-6, which can suppress the immune functions and initiate the defensive reactions, thus exerting bi-directional effects (18). IL-10, as another member in the interleukin family, suppresses the activity of natural killer cells, which aids the tumor cells to escape from the surveillance of immune system.
Under normal circumstances, IL-10 is at a low level, but after lobectomy, its concentration is elevated to a high level (19). The results of the present study showed that there were no statistically significant differences in a comparison of CRP, IL-6 and -10 levels before operation, but on the 3rd, 5th and 7 th days after operation, we found that the levels of CRP, IL-6 and -10 in patients in the observation group were significantly higher than those before the operation, but the levels in the observation were significantly lower than those in the control group, and the incidence rate of postoperative complications of patients in the observation was significantly lower than that in the control group $(\mathrm{P}<0.05)$. Compared with conventional thoracotomy, the minimally invasive segmentectomy generates fewer traumas and induces slighter stress responses in surgery, which may result in relatively slight reactions in the activated immune system. Thus, a release of cytokines, such as CRP, IL-6 and -10, may sustain a low level. Minimally invasive surgeries generate less postoperative pain, and scarcely hinder the recovery of patients, leading to a decrease in the incidence rate of complications.

The results of the present study showed that FVC, FEV1, FEV1/FVC and PEER of patients in the observation group after operation were significantly higher than those in the control group $(\mathrm{P}<0.05)$. This is because conventional thoracotomy may generate extensive injuries to muscles on the chest wall, severely affecting the activity of chest muscles. In addition, oppressions on lung can hardly be avoided in the operation, thus resulting in the contusion and edema in respiratory membrane. Moreover, postoperative cough and breath of patients may cause pain in incisions, while the practice of breath functions may decrease the compliance of patients. These factors contribute to a slow recovery in pulmonary function. With the assistance of video, microstructures in the thoracoscopic segmentectomy can be amplified. Thus, surgeons can avoid injuries to the lung, which can effectively protect the pulmonary function and promote the postoperative recovery of patients (20). With smaller incisions, slighter inflammatory responses and immunosuppression, and less effect on the immune functions, minimally invasive surgeries can significantly increase the survival rate and survival period of patients after operation.

In conclusion, compared with conventional thoracotomy, minimally invasive segmentectomy, with the advantages such as smaller incisions, rapid recovery of patient after operation, and less effect on the pulmonary function, is superior in safety and effectiveness. Thus, it is of important value in the surgical treatment of lung cancer at early stage.

\section{Acknowledgements}

Not applicable.

\section{Funding}

No funding was received.

\section{Availability of data and materials}

All data generated or analyzed during this study are included in this published article. 


\section{Authors' contributions}

CS contributed significantly to writing the manuscript and preoperative preparation. CZ detected and analyzed CRP, IL-6 and IL-10 levels in serum. WY and YS helped postoperative treatment and follow-up. ZZ contributed significantly to manuscript preparation, the conception of the study and statistical analysis. All authors read and approved the final manuscript.

\section{Ethics approval and consent to participate}

This study was approved by the Ethics Committee of The Affiliated Central Hospital of Qingdao University (Qingdao, China) and informed consents were signed by the patients and/ or guardians.

\section{Consent for publication}

Not applicable.

\section{Competing interests}

The authors declare that they have no competing interests.

\section{References}

1. Travis WD, Travis LB and Devesa SS: Lung cancer. Cancer 75: 191-202, 1995.

2. Del Vescovo V and Denti MA: microRNA and lung cancer. Adv Exp Med Biol 889: 153-177, 2015.

3. Howlader N, Noone AM, Krapcho M, Miller D, Bishop K, Kosary CL, Yu M, Ruhl J, Tatalovich Z, Mariotto A et al: SEER Cancer Statistics Review, 1975-2014. National Cancer Institute. Bethesda, Md: http://seer.cancer.gov/csr/1975_2014, based on November 2016 SEER data submission posted to the SEER web site, April 2017.

4. Puri V,Patel A, Majumder K, Bell JM, Crabtree TD, Krupnick AS, Kreisel D, Broderick SR, Patterson GA and Meyers BF: Intraoperative conversion from video-assisted thoracoscopic surgery lobectomy to open thoracotomy: A study of causes and implications. J Thorac Cardiovasc Surg 149: 55-61, 2015.

5. Yu PS, Capili F and Ng CS: Single port VATS: Recent developments in Asia. J Thorac Dis 8: 302-307, 2016.

6. Klapper J and D'Amico TA: VATS versus open surgery for lung cancer resection: Moving toward a minimally invasive approach. J Natl Compr Canc Netw 13: 162-164, 2015.

7. Cooper WA: Preface-molecular genetics of lung cancer. Transl Lung Cancer Res 4: 109, 2015.
8. Mohamed SAA, Mousa EM, Hamed AM, Amin SE and Abdel Aziz NMA: Utility of multidetector row computed tomography and virtual bronchoscopy in evaluation of hemoptysis due to lung cancer. Egypt J Chest Dis Tuberc 65: 279-287, 2016.

9. Lin L, Gu ZT, Chen WH and Cao KJ: Increased expression of the long non-coding RNA ANRIL promotes lung cancer cell metastasis and correlates with poor prognosis. Diagn Pathol 10: $14-21,2015$

10. Yu JB, Soulos PR, Cramer LD, Decker RH, Kim AW and Gross CP: Comparative effectiveness of surgery and radiosurgery for stage I non-small cell lung cancer. Cancer 121: 2341-2349, 2015.

11. Pan TW, Wu B, Xu ZF, Zhao XW and Zhong L: Video-assisted thoracic surgery versus thoracotomy for non-small-cell lung cancer. Asian Pac J Cancer Prev 13: 447-450, 2012.

12. Laursen L $\varnothing$, Petersen RH, Hansen HJ, Jensen TK, Ravn J, Kongea L: Video-assisted thoracoscopic surgery lobectomy for lung cancer is associated with a lower 30-day morbidity compared with lobectomy by thoracotomy. Eur J Cardiothorac Surg 49: 870-875, 2016.

13. Liu C, Li Z, Bai C, Wang L, Shi X and Song Y: Video-assisted thoracoscopic surgery and thoracotomy during lobectomy for clinical stage I non-small-cell lung cancer have equivalent oncological outcomes: A single-center experience of 212 consecutive resections. Oncol Lett 9: 1364-1372, 2015.

14. Gelalis ID, Arnaoutoglou CM, Politis AN, Batzaleksis NA, Katonis PG and Xenakis TA: Bacterial wound contamination during simple and complex spinal procedures. A prospective clinical study. Spine J 11: 1042-1048, 2011.

15. Li SF, Hu YW, Zhao JY, Ma X, Wu SG, Lu JB, Hu YR, Wang YC, Gao JJ, Sha YH, et al: Ox-LDL upregulates CRP expression through the IGF2 pathway in THP-1 macrophages. Inflammation 38: 576-583, 2015.

16. Waterland P, Ng J, Jones A, Broadley G, Nicol D, Patel H and Pandey S: Using CRP to predict anastomotic leakage after open and laparoscopic colorectal surgery: Is there a difference? Int J Colorectal Dis 31: 861-868, 2016.

17. Hunter CA and Jones SA: IL- 6 as a keystone cytokine in health and disease. Nat Immunol 16: 448-457, 2015.

18. Gomes M, Coelho A, Araújo A, Azevedo A, Teixeira ALO, Catarino R and Medeiros R: IL-6 polymorphism in non-small cell lung cancer: A prognostic value? Tumour Biol 36: 3679-3684, 2015.

19. Zhang YM, Mao YM and Sun YX: Genetic polymorphisms of IL-6 and IL-10 genes correlate with lung cancer in never-smoking Han population in China. Int J Clin Exp Med 8: 1051-1058, 2015.

20. Goto T, Kadota Y, Mori T, Yamashita S, Horio H, Nagayasu T and Iwasaki A: Video-assisted thoracic surgery for pneumothorax: Republication of a systematic review and a proposal by the guideline committee of the Japanese association for chest surgery 2014. Gen Thorac Cardiovasc Surg 63: 8-13, 2015.

This work is licensed under a Creative Commons Attribution-NonCommercial-NoDerivatives 4.0 International (CC BY-NC-ND 4.0) License. 